\title{
Tobacco cessation quitlines in North America: a descriptive study
}

\author{
Sharon E Cummins, Linda Bailey, Sharon Campbell, Carrie Koon-Kirby, Shu-Hong Zhu
}

Tobacco Control 2007;16(Suppl I):i9-i15. doi: 10.1136/tc. 2007.020370

See end of article for authors' affiliations

Correspondence to: Dr Shu-Hong Zhu, Cancer Center, 0905, University of California, San Diego, La Jolla, CA 92093-0905,

USA; szhu@ucsd.edu

Received 23 January 2007 Accepted 23 July 2007

\begin{abstract}
Background: Quitlines have become an integral part of tobacco control efforts in the United States and Canada. The demonstrated efficacy and the convenience of telephone based counselling have led to the fast adoption of quitlines, to the point of near universal access in North America. However, information on how these quitlines operate in actual practice is not often readily available.

Objectives: This study describes quitline practice in North America and examines commonalities and differences across quitlines. It will serve as a source of reference for practitioners and researchers, with the aim of furthering service quality and promoting continued innovation.

Design: A self administered questionnaire survey of large, publicly funded quitlines in the United States and Canada. A total of 52 US quitlines and 10 Canadian quitlines participated. Descriptive statistics are provided regarding quitline operational structures, clinical services, quality assurance procedures, funding sources and utilisation rates.

Results: Clinical services for the 62 state/provincial quitlines are supplied by a total of 26 service providers. Nine providers operate multiple quitlines, creating greater consistency in operation than would otherwise be expected. Most quitlines offer services over extended hours (mean 96 hours/week) and have multiple language capabilities. Most (98\%) use proactive multisession counselling - a key feature of protocols tested in previous experimental trials. Almost all quitlines have extensive training programmes ( $>60$ hours) for counselling staff, and over $70 \%$ conduct regular evaluation of outcomes. About half of quitlines use the internet to provide cessation information. A little over a third of US quitlines distribute free cessation medications to eligible callers. The average utilisation rate of the US state quitlines in the 2004-5 fiscal year was about $1.0 \%$ across states, with a strong correlation between the funding level of the quitlines and the smokers' utilisation of them $(r=0.74, p<0.001)$.

Conclusions: Quitlines in North America display core commonalities: they have adopted the principles of multisession proactive counselling and they conduct regular outcome evaluation. Yet variations, tested and untested, exist. Standardised reporting procedures would be of benefit to the field. Shared discussion of the rationale behind variations can inform future decision making for all North American quitlines.
\end{abstract}

programmes. ${ }^{14-16}$ The present study is the first attempt to systematically examine quitline practice since quitlines became universally available in the United States and Canada.

This paper reports on clinical, programmatic and administrative aspects of quitlines across the United States and Canada and looks at the resources allocated for these programmes. It aims to provide a general description of the current state of affairs for quitlines in North America and to serve as a reference for practitioners and researchers. It also identifies gaps in research and practice in order to further the quality of service and to encourage continued innovation and collaboration among existing and future quitlines. National Action Plan for Tobacco Cessation, ${ }^{9}$ and by federal funding to establish or expand state quitline services. ${ }^{10}$ In the United States, for example, a single toll-free number (800QUIT-NOW) has been created to serve as a national portal allowing tobacco users from any state to call for quitline services, usually provided free by the state from which the call originates.

This widespread adoption of quitlines has created a need for information on such issues as organisation of operations, the degree to which service protocols are guided by experimental results, quality control measures, effects of funding on organisation and service and level of utilisation by smokers. Surveys of quitlines have been conducted previously in the United States and Canada ${ }^{11-13}$ and some of the data have been used to encourage more states and provinces to establish quitlines as part of their comprehensive tobacco control

\section{METHODS}

\section{Participants}

This study focuses on state and provincial quitlines providing free tobacco cessation services to the general public. It does not include telephone programmes with access restricted to employees of certain companies or to members of certain health plans. At the time of this study, there were 62 state/ provincial quitlines operating in North America, which include all Canadian provinces $(n=10)$, all US states $(n=50)$, and one each in the District of Columbia and the territory of Puerto Rico. Most of these quitlines were funded by state/provincial

Abbreviations: NAQC, North American Quitline Consortium; NRT, nicotine replacement therapy; UCSD, University of California, San Diego 
agencies, the rest by federal agencies. The study survey was sent in October 2005 to quitline funders, who referred questions on clinical content to their contracted clinical providers (also referred to as quitline operators or vendors). Survey participation was voluntary. Data privacy policies were explained at the start of the survey. Participants were told that the data would be stored at the University of California, San Diego (UCSD), that the North American Quitline Consortium (NAQC) would own the data and be responsible for its proper use, and that all information regarding funding would be reported only in the aggregate, without identifying specific states/provinces.

\section{Survey materials}

The questionnaire was based on two earlier surveys, one conducted by UCSD in 2002 for the first North American Quitline Conference, ${ }^{11}$ and the other by NAQC in 2004 for regional meetings held by the Centers for Disease Control and Prevention (CDC) on tobacco cessation quitlines. ${ }^{12}$ Questions in the current survey covered operational issues such as hours and language capabilities, as well as counselling protocols, quality control procedures, staffing and training programmes, evaluation design and sources and levels of funding. This survey examined fiscal year 2004-5 (US July 2004-June 2005; Canada April 2004-March 2005). A major effort was made to create standard definitions of terms. For example, the term counselling was defined as "a person tailored, in-depth, motivational interaction that occurs between cessation specialist/counsellor and caller." Interactions that did not meet this standard were classified as brief. Counselling was subdivided into three categories: brief intervention ( $1-10$ minutes), single session (>10 minutes) and multiple session. Multiple session was further divided into reactive (smoker initiated follow-up) and proactive (counsellor initiated follow-up). The entire questionnaire can be viewed on the NAQC website: http://www. naquitline.org/assets/Survey_USA_2005.pdf.

\section{Procedure}

NAQC sponsored the survey and distributed it via email to the 62 state/provincial quitline funders in October 2005. Those not responding by December 2005 were contacted by NAQC staff, who encouraged survey completion and addressed any obstacles. All surveys were collected by April 2006. UCSD staff provided technical assistance to survey respondents regarding the questionnaire, entered the collected data for computer analysis and contacted respondents for clarification when necessary.
All quitlines responded to the survey. However, not all participating quitlines answered all questions. For example, some provided only information about operations. Therefore, the sample size for analyses varies by question, as shown in each table.

\section{Data analysis}

Data were aggregated by country, and standard deviations are shown for means, providing a measure of variation across quitlines. When needed, 95\% confidence intervals are provided for evaluation of significant differences. When calculating US state quitline utilisation rates, we used Current Population Surveys-Tobacco Use Supplement ${ }^{17}$ to obtain the smoking population for each state. All computations were done with SAS 9.1. ${ }^{18}$

\section{RESULTS}

\section{Service providers}

Clinical services for the 62 state/provincial quitlines are supplied by 26 service providers. Two thirds of these providers operate a single quitline. Nine of the organisations provide services to multiple states/provinces (range 2-14) with three organisations operating $44 \%$ of all quitlines (27/62).

\section{Hours of operation and language capacity}

Table 1 shows that quitline hours of operation (hours when staff answer the phone live) are extensive. For incoming calls, hours of operation range from 40 to 168 per week (that is, 24 hours per day, 7 days per week), with a mean of 96 hours per week. The availability of trained counsellors is somewhat lower, with the mean around 85 hours per week, although trained counsellors do staff some quitlines around the clock (168 hours per week). In addition to accepting incoming calls, many quitlines $(77.4 \%)$ accept fax referrals from healthcare providers and others. The referring agency obtains smokers' consent to share contact information with the quitline. Quitline counsellors then proactively contact these smokers and offer services, a process that integrates quitlines into the healthcare systems.

Language capacity (the presence of quitline counsellors who can provide the intervention without using a translation service) ranges from 1 to 8 , with the mean around 2 . US quitlines have a wider range of languages than have Canadian quitlines with the two most common languages offered being English and Spanish for US quitlines and English and French for Canadian quitlines.

\begin{tabular}{|lccc|}
\hline Table 1 & Quitline hours of operation and language capacity & \\
\hline & Total & United States & Canada \\
\hline Hours of operation (hours per week) & & & \\
For incoming calls & $n=60$ & $n=52$ & $n=8$ \\
Range & $40-168$ & $40-168$ & $56-168$ \\
Mean (SD) & $96.0(39.1)$ & $96.9(39.1)$ & $96.1(44.9)$ \\
Median & 86 & 96 & 77 \\
For counselling & $n=60$ & $n=52$ & $n=8$ \\
Range & $40-168$ & $40-168$ & $56-168$ \\
Mean (SD) & $85.3(27.8)$ & $85.8(27.5)$ & $85.6(34.3)$ \\
Median & 82 & 87 & 77 \\
Number of languages available & & & $n=8$ \\
Phone lines & $n=56$ & $n=48$ & $1-2$ \\
Range & $1-8$ & $1-8$ & $1.5(0.5)$ \\
Mean (SD) & $2.2(1.2)$ & $2.3(1.2)$ & 1.5 \\
Median & 2 & 2 & $n=8$ \\
Materials & $n=60$ & $n=52$ & $1-7$ \\
Range & $1-7$ & $1-7$ & $2.5(1.9)$ \\
Mean (SD) & $2.2(1.2)$ & $2.2(1.1)$ & 2 \\
Median & 2 & 2 & \\
\hline
\end{tabular}


Table 2 Services offered by quitlines

\begin{tabular}{|c|c|c|c|}
\hline & Total & \multirow{2}{*}{$\begin{array}{l}\text { United States } \\
\mathrm{n}=52\end{array}$} & \multirow{2}{*}{$\frac{\text { Canada }}{n=10}$} \\
\hline & $n=62$ & & \\
\hline & $\%(95 \% \mathrm{Cl})$ & $\%(95 \% \mathrm{Cl})$ & $\%(95 \% \mathrm{Cl})$ \\
\hline $\begin{array}{l}\text { Mailed self help materials } \\
\text { Telephone based services }\end{array}$ & $96.8(4.4)$ & $98.1(3.7)$ & $90.0(18.6)$ \\
\hline Proactive* with multiple sessions & $98.4(3.1)$ & 100 & $90.0(18.6)$ \\
\hline Reactive follow-up sessions & $37.1(12.0)$ & $28.8(12.3)$ & $80.0(24.8)$ \\
\hline Single session ( $>10$ minutes) & $66.1(11.8)$ & 61.5 (13.2) & $90.0(18.6)$ \\
\hline Brief session ( $1-10$ minutes) & $53.2(12.4)$ & $46.2(13.6)$ & $90.0(18.6)$ \\
\hline Recorded messages & $50.0(12.5)$ & $55.8(13.5)$ & $20.0(24.8)$ \\
\hline \multicolumn{4}{|l|}{ Internet based services } \\
\hline General quitline information & $53.2(12.4)$ & $53.8(13.6)$ & $50.0(30.1)$ \\
\hline Cessation information & $53.2(12.4)$ & $53.8(13.6)$ & $50.0(30.1)$ \\
\hline Self directed quit plan & $33.9(11.8)$ & $30.8(12.6)$ & $50.0(30.1)$ \\
\hline Automated email messages & $21.0(10.1)$ & $13.5(9.3)$ & $60.0(30.4)$ \\
\hline Chat rooms & $17.7(9.5)$ & $11.5(8.7)$ & $50.0(30.1)$ \\
\hline Interactive counselling & $29.0(11.3)$ & 26.9 (12.1) & $40.0(30.4)$ \\
\hline \multicolumn{4}{|l|}{ Cessation medications } \\
\hline Free medications & & $34.6(12.9)$ & 0 \\
\hline Discounted medications & & $9.6(8.0)$ & 0 \\
\hline Vouchers & & $23.1(11.5)$ & 0 \\
\hline Any of the above & & $55.8(13.5)$ & 0 \\
\hline
\end{tabular}

\section{Services offered}

Table 2 presents data on types of services available from quitlines in the United States and Canada in fiscal year 2004-5. It shows that all 52 US quitlines and 9 Canadian quitlines offered multisession, proactive phone counselling. In addition, some quitlines provided follow-up sessions in a reactive mode: clients were encouraged to call back as needed, outside their scheduled proactive sessions. Canadian quitlines appear to have adopted this policy more consistently than US quitlines.

Besides providing multiple counselling sessions, $61.5 \%$ of US quitlines and $90 \%$ of Canadian quitlines also provided single counselling sessions (>10 minutes) to some callers without further follow-up. Moreover, over half of the quitlines provided brief interventions ( $\leqslant 10$ minutes) to callers who simply wanted questions answered without further therapeutic discussion. And about half of the quitlines provided recorded cessation information that callers could access outside normal operating hours.

In addition to phone based services, about half of the quitlines also provided cessation related services via the internet. These services varied from website information about quitline operation (for example, phone numbers and hours) to interactive web based counselling, available from about $29 \%$ of quitlines.

There were variations in methods of incorporating cessation medications (patch, gum, Zyban, etc) into quitline services. No quitlines in Canada directly or indirectly provided medications. In the United States, $34.6 \%$ of state quitlines mailed free medications to eligible callers, and a few others provided discounts or vouchers. In some cases, the mailing of free medications was intended to be ongoing; in others, it was limited to the 2004-5 fiscal year. Overall, more than half of state quitlines $(55.8 \%)$ were providing either free or discounted cessation medication.

\section{Eligibility for quitline services}

Many US quitlines employed eligibility criteria for clients wishing to receive free counselling or medications. No Canadian quitlines reported restricting services. Nearly $62 \%$ of US quitlines used one or both of the following restrictions: (1)

Table 3 Counselling protocols: standard and specialised

\begin{tabular}{llll} 
& Total & United States & Canada \\
\hline Standard protocol & $\mathrm{n}=61$ & $\mathrm{n}=51$ & $\mathrm{n}=10$ \\
& Mean (SD) & Mean (SD) & Mean (SD) \\
Number of sessions & $5.1(1.3)$ & $5.0(1.2) \dagger$ & $7.0(1.0) \dagger$ \\
1st session length (minutes) & $28.3(9.8)$ & $30.2(9.4)$ & $18.1(2.7)$ \\
Follow-up session length (minutes) & $16.1(8.3)$ & $17.2(8.6)$ & $9.6(1.5)$ \\
& $\%$ & & $\%$ \\
Specialised protocols & $\%$ & $\%$ & $\%$ \\
Pregnant women & $88.5(8.0)$ & $90.2(8.2)$ & $80.0(24.8)$ \\
Smokeless tobacco & $60.7(12.3)$ & $70.6(12.5)$ & $10.0(18.6)$ \\
Ethnic populations & $42.6(12.4)$ & $51.0(13.7)$ & 0 \\
Youths (12-17 years) & $39.3(12.3)$ & $45.1(13.7)$ & $10.0(18.6)$ \\
Multiple addictions & $16.4(9.3)$ & $15.7(10.0)$ & $20.0(24.8)$ \\
Young adults (18-24 years) & $13.1(8.5)$ & $15.7(10.0)$ & 0 \\
LGBT & $9.8(7.5)$ & $11.8(8.9)$ & 0 \\
Chronic mental illness & $9.8(7.5)$ & $9.8(8.2)$ & $10.0(18.6)$ \\
Older adults (55+ years) & $8.2(6.9)$ & $9.8(8.2)$ & 0 \\
\hline
\end{tabular}

"Number of sessions refers to intended sessions prescribed by the protocol, not actual number of sessions delivered. + Numbers for this item are United States $=49$ and Canada $=3$.

łLesbian, gay, bisexual, transgendered. 
Table 4 Counselling quality control activities

\begin{tabular}{|c|c|c|c|}
\hline & Total & United States & Canada \\
\hline \multicolumn{3}{|l|}{ Class training } & $n=9$ \\
\hline$\%$ quitlines ${ }^{*}(95 \%$ Cl) & $96.6(4.6)$ & $96.0(5.4)$ & 100 \\
\hline Mean (SD) hours & $32.9(17.9)$ & 35.8 (17.4) & $19.2(13.5)$ \\
\hline \multicolumn{4}{|l|}{ Role playing } \\
\hline$\%$ quitlines* $(95 \% \mathrm{Cl})$ & $93.2(6.4)$ & $92.0(7.5)$ & 100 \\
\hline Mean (SD) hours & $8.5(12.3)$ & $8.6(13.7)$ & $8.0(3.0)$ \\
\hline \multicolumn{4}{|l|}{ Call shadowing } \\
\hline$\%$ quitlines* $(95 \% \mathrm{Cl})$ & $93.2(6.4)$ & $94.0(6.6)$ & $88.9(20.5)$ \\
\hline Mean (SD) hours & $19.4(18.8)$ & $21.4(20.0)$ & $9.4(2.7)$ \\
\hline \multicolumn{4}{|l|}{ Other } \\
\hline$\%$ quitlines* $(95 \% \mathrm{Cl}$ ) & $81.4(9.9)$ & $82.0(10.7)$ & $77.8(27.2)$ \\
\hline Mean (SD) hours & $15.4(5.9)$ & $14.6(6.0)$ & $20.2(2.0)$ \\
\hline \multicolumn{4}{|l|}{ Continuing education } \\
\hline$\%$ quitlines $(95 \% \mathrm{Cl}$ ) & $96.6(4.6)$ & $96.2(5.4)$ & 100 \\
\hline Modal hours/year & $21-30$ & $21-30$ & $21-30$ \\
\hline Counsellor supervision & $n=61$ & $n=52$ & $n=9$ \\
\hline & $\%(95 \% \mathrm{Cl})$ & $\%(95 \% \mathrm{Cl})$ & $\%(95 \% \mathrm{Cl})$ \\
\hline Individual supervision & $82.0(9.6)$ & $78.8(11.1)$ & 100 \\
\hline Group supervision & $78.6(10.3)$ & $90.4(8.0)$ & $11.1(20.5)$ \\
\hline Peer supervision & $54.1(12.5)$ & $50.0(13.6)$ & 77.7 (27.2) \\
\hline Review taped call & $68.9(11.6)$ & $75.0(11.8)$ & $33.3(30.8)$ \\
\hline Live call monitor & $85.2(8.9)$ & $82.6(10.3)$ & 100 \\
\hline
\end{tabular}

counselling may be contingent on smokers' readiness to quit (reported by $81 \%$ of US quitlines that use restrictions), and (2) multiple follow-up sessions may be contingent on insurance status. Of US quitlines providing free medications, $61.1 \%$ reported using insurance status to screen for eligibility for medications (data not presented in table). Generally speaking, lack of insurance entitled callers to greater service.

\section{Telephone counselling protocols}

Table 3 displays summary data regarding counselling protocols. All responding quitlines used standardised, multisession protocols for typical adult clients. The mean number of planned sessions was around five. Only three Canadian quitlines provided information about number of sessions, which averaged seven.

The first counselling session typically was designed to be longer than the follow-up sessions, as in most experimental studies to date. ${ }^{4}{ }^{19}$ All quitlines reported that their counselling addressed motivation, use of quit aids, planning and coping strategies, setting a quit date, and development of skills to prevent relapse.

The survey defined specialised counselling protocols as "different from standard counselling and going beyond the tailoring that counsellors normally do for the individual client." Most quitlines had such protocols for pregnant smokers $(88.5 \%)$ and smokeless tobacco users $(60.7 \%)$. Some also had specialised protocols for youths (12-17-year-olds, 39.3\%), for ethnic populations $(42.6 \%)$, and for other specific populations, such as people with chronic mental illness.

\section{Counselling quality control activities}

Table 4 provides results about procedures used to maintain the quality of quitline services. A consistent pattern of training

Table 5 Quitline funders and levels of funding

\begin{tabular}{|c|c|c|}
\hline \multirow[b]{3}{*}{ Quitline funders } & \multirow{2}{*}{$\begin{array}{l}\text { United States } \\
n=52\end{array}$} & \multirow{2}{*}{$\begin{array}{l}\text { Canada } \\
n=8\end{array}$} \\
\hline & & \\
\hline & $\%$ & $\%$ \\
\hline Federal & $67.3(12.8)$ & $37.5(33.6)$ \\
\hline State/province & $71.2(12.3)$ & 62.5 (33.6) \\
\hline$M S A^{*}$ funds & $48.1(13.6)$ & - \\
\hline Tobacco tax & $17.3(10.3)$ & 0 \\
\hline Other & 7.7 (7.3) & 0 \\
\hline Local & $3.8(5.2)$ & $12.5(22.9)$ \\
\hline Healthcare organisation & $3.8(5.2)$ & 0 \\
\hline Insurance company & $5.8(6.4)$ & 0 \\
\hline Charitable group & $3.8(5.2)$ & 0 \\
\hline NGO† & $3.8(5.2)$ & $12.5(22.9)$ \\
\hline Employer organisation & 1.9 (3.7) & 0 \\
\hline Other & $5.8(6.4)$ & $12.5(22.9)$ \\
\hline & $\mathrm{n}=44$ & $n=6$ \\
\hline Median & $\$ 621696$ & \$C204 893 \\
\hline Range & $\$ 40000-$ & \$C59 920-\$C512 134 \\
\hline
\end{tabular}

*Tobacco industry Master Settlement Agreement.

†Non-governmental organisation. 
activities emerged, although there were differences in the amount of time devoted to each training activity. On average, intervention staff received over 60 hours of initial training, mostly involving classroom instruction, with some role play and call shadowing with veteran counsellors (the "other" category included mostly administrative and technological training). In addition to initial training activities listed, over $95 \%$ of US and Canadian quitlines had continuing education programmes for their counsellors. Table 4 also shows counselling supervision strategies. Common strategies include individual supervision $(82.0 \%$ of quitlines) and live call monitoring $(85.2 \%$ of quitlines).

\section{Programme evaluation}

Over $70 \%$ of US quitlines reported conducting programme evaluations with callers. Of the quitlines involved in formal evaluation, almost all collected quit outcomes (97.7\%) and client satisfaction data (95.3\%). Fewer (62.8\%) collected data regarding clients' evaluation of counsellors. Most programme evaluation activities in Canada (87.5\%) and about half in the United States $(51.2 \%)$ were conducted by organisations independent of the service providers.

\section{Funding sources and levels}

Table 5 summarises information about funding sources for current quitlines in North America. Federal, state/provincial and local governments have all contributed funding to existing quitlines. It should be emphasised that states/provinces provided the bulk of the funding. In the United States, especially, the states drew quitline funding from multiple sources. Many US states used funds from the tobacco industry Master Settlement Agreement to establish quitlines (48.1\%), and some state quitlines were funded by special cigarette taxes instituted in individual states $(17.3 \%)$. The average level of funding for quitline services $(n=44)$ in US states for fiscal year 2004-5 was about $\$ 620000$, with a range from $\$ 40000$ to over $\$ 4$ million. Only six Canadian quitlines reported their funding levels, and their range appeared narrower, with the median around \$C200 000.

\section{Quitline utilisation rates in the United States}

Figure 1 provides data on the percentage of smokers in each US state who called state quitlines, as a function of state spending per smoker. There was considerable variability across states in both utilisation rate (range $0.01 \%-4.28 \%$ ) and spending per smoker (range \$0.07-\$8.32 spent per smoker). There was a strong positive correlation between the money spent on quitlines per smoker in the state and the percentage of smokers in that state calling the quitline $(\mathrm{r}=0.74, \mathrm{p}<0.001)$. Giving equal weight to each state (that is, disregarding population size), the average utilisation rate across the states was $0.99 \%$ in 2005 , with an average of $\$ 1.77$ spent per smoker. Canadian data

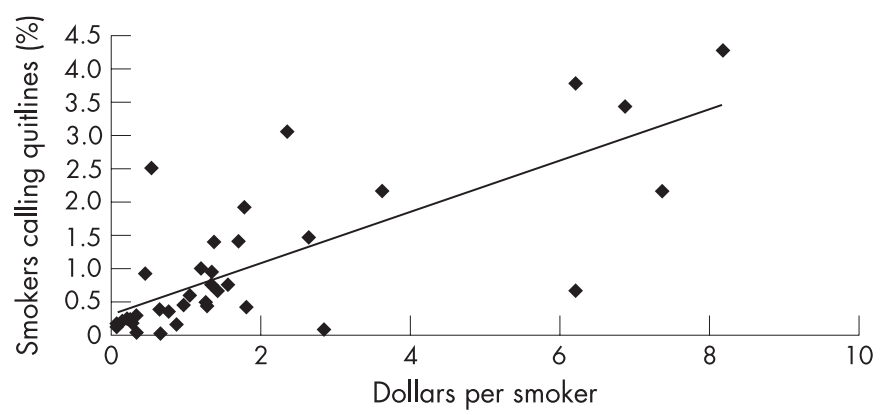

Figure 1 Rate of utilisation and spending in fiscal year 2004-5 ( $n=36)$. were not included because only three provinces provided both expenditure and utilisation data.

\section{DISCUSSION}

Survey results showed that state and provincial quitlines in North America share common elements of practice including core service activities and commitment to quality assurance. Results revealed variations among the quitlines regarding funding, services and utilisation. Differences among quitlines may reflect specific contexts of given tobacco control programmes (for example, budget constraints), the pursuit of new ideas (for example, internet based intervention) or variations in implementation (for example, number of counselling sessions). Examination of these commonalities and differences reveals knowledge gaps and may further quitline evolution and impact.

One commonality is that almost all state and provincial quitlines endorsed the use of multisession, proactive counselling. This treatment protocol has the strongest evidence base; proactive counselling is the key feature of the protocols tested in experimental studies and proved effective. ${ }^{1-4}{ }^{20}$ The adoption of multisession, proactive counselling by the many different funders and service providers is clearly a successful case of disseminating research results to practice. ${ }^{1-4}$ Single session counselling (when it adheres to a comprehensive protocol) has also been proved to be effective, and most quitlines offered this service as well. ${ }^{4}$

Counselling was universally characterised as addressing motivation, use of quit aids, planning and coping strategies, setting a quit date, and development of skills to prevent relapse. Despite the general agreement on counselling content, it remains unclear exactly what transpires in each quitline's counselling sessions. With few exceptions, ${ }^{19}{ }^{21}$ quitline operators have not detailed their counselling protocols in public documents. An additional issue hampering accurate comparison of interventions is that implementation of a protocol often deviates from the intended intervention. For example, two quitlines may adopt the same standardised protocol, with the same number of proactive follow-up sessions prescribed, and yet differ in the average number of follow-up sessions delivered. Deviations from the intended intervention might arise as a result of differences in staffing levels or in persistence of counsellors attempting to reach smokers who miss appointments. In short, the actual delivery of counselling may deviate from a stated standardised protocol in many important ways, leaving a significant knowledge gap in quitline operation. The quitline community (through NAQC) has embraced the standardisation of intake data through its recommended minimal data set. ${ }^{22}$ It would likewise be useful to work towards a consistent format for documenting the delivery of counselling.

Most quitlines appear to have quality control built into their operations. Quality of service starts with training, and the survey results indicate that most quitlines provide lengthy training before counselling staff begin taking clients. Most quitlines augment the standard didactic classroom hours with additional modalities of learning such as role playing and shadowing, providing opportunities to develop the nuances of counselling. Supervision ranges from daily, informal peer contacts or weekly groups to quarterly, individual supervision sessions. Most quitlines also have formal continuing education programmes to ensure that counsellors stay up to date on developments in tobacco control. This considerable investment in counsellor training and supervision allows quitlines to be staffed by para-professionals and professional counsellors

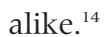

Most quitlines continuously assess quality of work through evaluation of clients' quitting outcomes. Whereas details of 
evaluation procedures differ across quitlines (another place where standardised reporting would be useful), the policy of ongoing outcome evaluation is prudent because treatment effects in real world application of clinically proved intervention protocols may not equate with effects observed in clinical studies. For example, the treatment effect may decay over time as counsellors drift from the rigour of the intervention delivered in the clinical trials. Emphasis on continuous performance assessment keeps programmes accountable and ensures the ongoing effectiveness of the intervention.

Quitlines provide extensive coverage across area, time and language. This is economically feasible because quitlines are centralised operations: a few counsellors on duty can cover a large geographic area and several different languages. It is interesting to note that compared to unpublished data from the 2002 quitline survey, ${ }^{11}$ the hours of operation for quitlines in North America in 2005 have increased, as have the numbers of quitlines providing counselling "in language" without the use of a translation service. These positive trends reflect quitline operators' efforts to increase accessibility and thereby improve service.

Survey results show that quitlines are largely supported by state and provincial funding, with additional federal monies. On average $1 \%$ of smokers in the United States utilised quitlines in the fiscal year 2004-5, although utilisation rates ranged from $0.01 \%$ to $4.3 \%$. As figure 1 shows, quitline utilisation rates were directly related to funding levels. Interestingly, however, some quitlines with lower levels of funding managed to reach greater proportions of smokers. The current survey did not examine the important topic of quitline promotion. Although it is well known that mass media have been the major channel of promotion for quitlines, ${ }^{23-25}$ more studies on various promotional strategies and their effectiveness are needed. ${ }^{26}$

Variations in practice naturally arise among entities that differ from one another in terms of geographic areas covered, populations served and structure (for example, medical centre/ university based, commercial enterprises, etc). These variations are important in two ways. Firstly, they provide the contextual information necessary to make sense of data provided by each quitline. Secondly, they represent alternate approaches that can fuel future research. One example is the use of eligibility criteria for providing counselling. Some quitlines counsel only smokers who are ready to quit-that is, they reserve the most intensive and costly intervention for clients who state a desire to take action. Restricting eligibility affects the context in which clients receive the counselling intervention and thereby affects the absolute quit rates. Knowing whether counselling is restricted, and to whom, is important in assessing data on success rates, especially if the data are being used to compare service providers. ${ }^{14}$ At the same time, the use of readiness to quit as a criterion for counselling is consistent with the evidence base, since most randomised trials of quitlines have recruited smokers who were ready to quit. The alternate approach would be to counsel all callers, regardless of their stated readiness to take action. Whether quitlines should allocate counselling resources according to readiness to change (which is more like a stepped care approach) or counsel every smoker without regard to readiness is still an open question whose answer has both scientific and practical implications.

Variations can reveal values underlying decisions about scope of practice, resource allocation, and services beyond the current evidence base. One example is the provision of free cessation medications. In 2004-5, one third of US quitlines reported providing nicotine replacement therapy (NRT), and another $20 \%$ provided vouchers or discounts. Both medication and telephone counselling are effective; providing both permits "one-stop shopping" and decreases barriers to quitting.

\section{What this paper adds}

- This paper reports on the first survey to systematically examine quitline practice since quitlines became universally available in the United States and Canada.

- It provides information about operational issues (such as hours and language capacities), service organisation, counselling protocols, quality control procedures, staffing and training programmes, evaluation design, and sources and levels of funding for 62 statewide and province-wide quitlines.

Offering free medication has been shown to markedly increase the number of smokers calling the quitline. ${ }^{27-29}$ Given these advantages, it is somewhat surprising that only one third of US quitlines provide this service. One issue may involve whether provision of medications falls within quitlines' scope of work or is the responsibility of health plans. Public funding for tobacco control is limited, and many quitlines opt to reserve their resources for counselling. Several service providers are conducting studies to examine issues (for example, cost) associated with providing free medication via quitline. ${ }^{30}$

Another promising direction is the use of internet based interventions. Only about half of quitlines use the internet to provide cessation information, and fewer still offer such components as chat rooms or interactive counselling. Given the internet's potential for reaching very large numbers of smokers, the use of internet interventions and the integration with telephone counselling are promising areas of exploration. Several quitline operators are currently conducting studies of internet based interventions ${ }^{31}$ to provide stronger empirical evidence of efficacy.

Some limitations of the survey itself need to be acknowledged. Although this survey provided more specific data than have previous surveys, ${ }^{11}{ }^{12}$ results are still limited by respondents' interpretations, and there were questions left blank by respondents. We have identified in the tables the effective sample size for each question. Despite those limitations, the overall data patterns clearly show where quitlines share common practices and where they diverge. However, the survey did not examine reasons for variations. This leaves us to raise questions and speculate about implications.

It should be mentioned that the unit of analysis in reporting results is the quitline. As a result, descriptions are weighted towards the practices of the nine organisations that provide service to multiple states or provinces. Changes in the number of service providers would inevitably affect the variability of practice. One result of the limited variability is greater consistency across quitlines in areas such as quality control or adherence to a minimal data set for screening and evaluation. ${ }^{22}$

\section{CONCLUSION}

The quitline milieu is dynamic, richly varied and marked by innovation. Multisession, proactive counselling is offered by over $98 \%$ of quitlines, and there is a strong shared commitment to training, supervision and ongoing evaluation of service. The natural variations in practice provide a springboard for discussion. Quitline researchers continue to gather empirical evidence for services. Survey results reveal gaps in our understanding of contextual issues and in the implementation of various protocols; standardised reporting would be useful in these areas. Results also reveal a knowledge gap regarding the rationale for various quitline practices, an area that would benefit from further discussion. Collaboration and cooperation 
among quitline stakeholders have always been strengths, which bodes well for the future of this kind of discussion.

\section{ACKNOWLEDGEMENTS}

We are grateful for the time that quitline funders and service providers spent answering the survey questionnaire. The authors also wish to thank Dr Shiushing Wong for statistical assistance.

\section{Authors' affiliations}

Sharon E Cummins, Carrie Koon-Kirby, Shu-Hong Zhu, University of

California, San Diego, CA, USA

Linda Bailey, North American Quitline Consortium

Sharon Campbell, Centre for Behavioural Research and Program Evaluation, Waterloo, Ontario, Canada

Funding: This study was supported by a supplemental grant to the Cancer Center at University of California, San Diego: National Cancer Institute Grant No 5P30CA23100-22 (to SHZ), and by a grant from the American Legacy Foundation (to North American Quitline Consortium). All authors of this paper are involved with quitline research or service and receive funding from federal and state/provincial governments.

Competing interests: none.

Ethical approval not required.

\section{REFERENCES}

1 Lichtenstein E, Glasgow RE, Lando HA, et al. Telephone counseling for smoking cessation: rationales and meta-analytic review of evidence. Health Educ Res 1996; 11:243-57.

2 Stead LF, Perera R, Lancaster T. A systematic review of interventions for smokers who contact quitlines. Tobacco Control 2007;16(Suppl I):i3-8.

3 Orleans CT, Schoenbach VJ, Wagner EH, et al. Self-help quit smoking interventions: effects of self-help materials, social support instructions, and telephone counselling. J Consult Clin Psychol 1991;59:439-48.

4 Zhu SH, Stretch V, Balabanis M, et al. Telephone counseling for smoking cessation: effects of single-session and multiple-session interventions. J Consult Clin Psychol 1996:59:439-48.

5 Ossip-Klein DJ, Giovino GA, Megahed N, et al. Effects of a smoker's hotline: results of a 10-county self-help trial. J Consult Clin Psychol 1991;59:325-32.

6 Anderson CM, Zhu SH. Tobacco quitlines: looking back and looking ahead. Tobacco Control 2007;16(Suppl I):i81-6.

7 Fiore MC, Bailey WC, Cohen SJ, et al. Treating tobacco use and dependence: a clinical practice guideline. Rockville, MD: US Department of Health and Human Services, 2000.

8 Hopkins DP, Briss PA, Ricard CJ, Task Force on Community Preventive Services, et al. Reviews of evidence regarding interventions to reduce tobacco use and exposure to environmental tobacco smoke. Am J Prev Med 2001;20(suppl 2):16-66

9 Fiore MC, Croyle RT, Curry SJ. Preventing 3 million premature deaths and helping 5 million smokers quit: a national action plan for tobacco cessation. Am J Public Health 2004;94:205-10.

10 US Department of Health and Human Services. HHS announces national smoking cessation quitline network. Available at http://www.hhs.gov/news/ press/2004pres/20040203.html. Accessed 5 Jun 2007.
11 Zhu SH. A survey of quitlines in North America, Presented at the Northern American Conference of Smoking Cessation Quitlines, May 2002. Phoenix, Arizona, USA.

12 North American Quitline Consortium. NAQC Survey of Quitlines, 2004.Available at http://naquitline.org/ index.asp?dbib = 5\&dbsection = research. Accessed 5 Jun 2007.

13 Keller PA, Bailey LA, Koss KJ, et al. Organization, financing, promotion, and cost of U.S. quitlines, 2004. Am J Prev Med 2007;32:32-7. Epub 20 Dec 2006.

14 Centers for Disease Control and Prevention. Telephone quitlines: a resource for development, implementation, and evaluation. Atlanta, GA: US Department of Health and Human Services, Centers for Disease Control and Prevention, National Center for Chronic Disease Prevention and Health Promotion, Office on Smoking and Health, final ed, Sept, 2004.

15 Ossip-Klein DJ, Mclntosh S. Quitlines in North America: evidence base and applications. Am J Med Sci 2003;326:201-5.

16 Borland R, Segan CJ. The potential of quitlines to increase smoking cessation. Drug Alcohol Rev 2006;25:73-8.

17 National Cancer Institute, Cancer Control and Population Sciences. Tobacco use supplement to the Current Population Survey, 2003. Available at http:// riskfactor.cancer.gov/studies/tus-cps/ Accessed 29 Nov, 2006.

18 SAS Institute Inc. SAS 9.1 Language reference: concepts. Cary, NC: SAS Institute, Inc, 2004.

19 Zhu SH, Tedeschi G, Anderson CM, et al. Telephone counseling for smoking cessation: What's in a call? JCD 1996;75:93-102.

20 McAlister AL, Rabius V, Geiger A, et al. Telephone assistance for smoking cessation: one year cost effectiveness estimations. Tobacco Control 2004;13:85-6.

21 American Legacy Foundation and American Cancer Society. Great Start quitline counseling protocol. Available at http://www.naquitline.org/ downloadfile.asp?\&Attachment = True\&MyPath =/downloads/topics/ \&MyFile=9_great_start_protocol.doc. Accessed 3 Jan, 2007.

22 Campbell SH, Ossip-Klein D, Bailey L, et al. Minimal dataset for quitlines: a best practice. Tobacco Control 2007;16(Suppl I):i16-20.

23 Zhu SH, Anderson CM, Johnson CE, et al. A centralised telephone service for tobacco cessation: the California experience. Tobacco Control 2000;9:48-55.

24 Miller CL, Wakefield M, Roberts L. Uptake and effectiveness of the Australian telephone Quitline service in the context of a mass media campaign. Tobacco Control 2003;12(Suppl 2):53-8.

25 Owen L. Impact of a telephone helpline for smokers who called during a mass media campaign. Tobacco Control 2000;9:148-54.

26 Chin NP, Ossip-Klein DJ, Padmore T, et al. Quitline counselors' perspectives on engaging underserved smokers in telephone counseling. Poster presented at the 13 th Annual Meeting of the Society for Research on Nicotine and Tobacco, 2124 Feb 2007. Austin, TX, USA.

27 An LC, Schillo BA, Kavanaugh AM, et al. Increased reach and effectiveness of a statewide tobacco quitline after the addition of access to free nicotine replacement therapy. Tobacco Control 2006;15:286-93.

28 Grigg M, Waa A. Improving effectiveness of smoking cessation helplines through the provision of subsidised nicotine replacement therapy: a longitudinal study. Conference presentation. Wellington New Zealand: The Quit Group and Health Sponsorship Council, 2003.

29 Cummings KM, Fix B, Celestino P, et al. Reach, efficacy, and cost-effectiveness of free nicotine medication giveaway programs. J Public Health Management and Practice 2006;12:37-43.

30 Fellows JL, Bush T, McAfee T, et al. Cost effectiveness of the Oregon quitline "free patch initiative". Tobacco Control 2007;16(Suppl I):i47-52.

31 Rabius V, Pike KJ, Geiger A, et al. American Cancer Society's QUITLINK: A randomized trial of Internet assistance for smoking cessation. Paper presented at the 13th World Conference on Tobacco or Health, 12-15 Jul 2006. Washington DC, USA. 